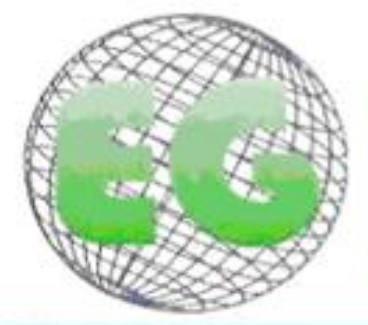

\title{
REVISIONES
}

\section{Mensajes de texto para el control glucémico en adultos con diabetes tipo 2: revisión sistemática}

Text messaging interventions to glycemic control in type 2 diabetes adults: systematic review

\section{*Valles Ortiz, Patricia M **Miranda Felix, Patricia ***García Sosa, Elia S}

\author{
*Estudiante de Doctorado en Ciencias de Enfermería, Facultad de Enfermería de la Universidad \\ Autónoma de Nuevo León. E-mail: pvalles@uacj.mx **Profesor de Tiempo Completo, Programa de \\ Enfermería del Instituto de Ciencias Biomédicas de la Universidad Autónoma de Ciudad Juárez. \\ ***Profesor de la Escuela de Enfermería de la Universidad Autónoma de Sinaloa. México.
}

\section{Palabras Clave: diabetes tipo 2; hemoglobina glucosilada; mensajes de texto; teléfono móvil Keywords: type 2 diabetes; glycosylated hemoglobin; text messaging; mobile phone}

\section{RESUMEN}

Objetivo: Evaluar la evidencia disponible sobre la efectividad de las intervenciones que han utilizado mensajes de texto para la disminución de los niveles de hemoglobina glucosilada en el control glucémico en personas con DT2

Métodos: Se realizó una revisión sistemática en las bases de datos MEDLINE, Elsevier, Springer, DARE, CINAHL, Scopus, y Open Access, se identificaron ocho artículos que cumplieron con los criterios de elegibilidad para evaluar el uso de los mensajes de texto y los niveles de HbA1c.

Resultados: El envío de mensajes de texto a través de teléfono móvil mostró una disminución de los niveles de hemoglobina glucosilada en pacientes adultos con DT2

Conclusiones: La utilización de mensajes de texto en la atención de los pacientes con DT2, se presenta como una tecnología factible y bien aceptada sin embargo los estudios señalaron que la evidencia aún no es concluyente en la mejoría del control glucémico.

\section{ABSTRACT}

Objective: The aim of this systematic review was to evaluate the effectiveness interventions that have used text messaging in the reduction of glycosylated hemoglobin levels in type 2 diabetes patients.

Methods: a systematic review of articles published in the MEDLINE database, Elsevier, Springer, DARE, CINAHL, Scopus, and Open Access, eight interventions studies that met the inclusion criteria, were identified. 
Results: Intervention using short message services of cellular phones improved $\mathrm{HbA} 1 \mathrm{c}$ levels and improved the metabolic control in T2D adults.

Conclusions: The use of text messaging in the management of $\mathrm{T} 2 \mathrm{D}$, is presented as a suitable, practical, and well accepted technology, it has strong potential for providing effective, ongoing support in the future. However the research studies, showed that the evidence is not yet conclusive to the improvement in glycemic control.

\section{INTRODUCCIÓN}

La diabetes se ha identificado como un problema de salud que afecta a las poblaciones de altos, medios y bajos ingresos. El costo asociado a su tratamiento y complicaciones representa la carga más grande no solo para los servicios de salud sino también para los enfermos y sus familias ${ }^{1}$. La Organización Mundial de la Salud (OMS) calcula que el número de personas con diabetes en el mundo es de 347 millones y pronostica que esta cifra se duplicará para el año 2030. Señala además que el $90 \%$ de los casos corresponden a diabetes tipo 2 (DT2) datos que la convierten en una de las principales amenazas para la salud en el siglo $21^{2}$. En México la DT2 se considera como el principal problema de salud entre su población. En la Encuesta Nacional de Salud $2012^{3}, 6.4$ millones de personas refirieron haber sido diagnosticadas con diabetes. La DT2 está considerada como la primera causa de muerte y es la causa más frecuente de incapacidad prematura, ceguera, insuficiencia renal terminal y de amputaciones no traumáticas. Además con frecuencia es una enfermedad que se diagnostica hasta que las complicaciones se han hecho presentes ${ }^{4,5}$.

La DT2 es ante todo una enfermedad de automanejo, su tratamiento se asocia en gran medida con la modificación de conductas. A partir del diagnóstico las personas con DT2 se convierten en las principales responsables del manejo de su enfermedad y por ende de llevar a cabo las acciones farmacológicas y no farmacológicas que les son prescritas como parte del plan terapéutico ${ }^{6}$. La realización de estas acciones requiere del apoyo permanente mediante consejería y/o asesoría por parte de los profesionales de salud sobre la dieta, la actividad física, la toma de medicamentos orales o aplicación de insulina y el automonitoreo de glucosa en sangre capilar para obtener un control óptimo del padecimiento y evitar o retardar complicaciones. Debido a la prevalencia de la DT2 es necesaria la implementación de estrategias alternativas que aseguren mejoras a largo plazo $0^{7-9}$.

La telefonía móvil es una de las tecnologías más extendida por todo el mundo, es por lo tanto esperado que su uso esté siendo considerado para incluirse en las aplicaciones médicas y se desarrollen programas específicos sobre todo para favorecer el autocontrol de enfermedades crónicas como la diabetes ${ }^{10-12}$.

Para la revisión de la literatura se utilizó la metodología de los siete pasos de Cooper $^{13}$. La evidencia disponible señala que las tecnologías de la información y comunicación (TIC's) han posibilitado el monitoreo a distancia de los resultados de diversas variables fisiológicas favoreciendo un mejor manejo a los pacientes con DT2, obteniendo beneficios como la disminución de las visitas a centros hospitalarios y recibiendo apoyo en tiempo real para su condición de salud. El uso de esta tecnología permite el envío de recordatorios para el automanejo en el que se incluye el monitoreo de glicemia capilar, los niveles de hemoglobina glucosilada (HbA1c) y mensajes educativos y motivacionales para el autocuidado ${ }^{14,15}$. A pesar de señalarse en algunos estudios resultados modestos de la utilización de tecnologías de la 
información para el cuidado de la DT2 ${ }^{16}$ las intervenciones basadas en el envío de mensajes de texto para el autocontrol de los pacientes con DT2 describieron una mejora en los niveles de HbA1c y destacaron como beneficio adicional el establecer o mejorar la comunicación entre el personal de salud y el paciente ${ }^{17,18}$.

La relativa facilidad con la que puede ser enviado un mensaje de texto o short message service (SMS) por sus siglas en Inglés, convierten la utilización del teléfono móvil en un medio de comunicación ideal para el intercambio de información entre el paciente con DT2 y el personal de salud ${ }^{18,19}$. Son pocos los estudios publicados hasta la fecha que han examinado si la telefonía móvil es una herramienta eficaz para mejorar el control glucémico o si el apoyo a través de mensajes de texto ofrece suficientes ventajas sobre el seguimiento del automanejo para generalizar su utilización. El uso de los sistemas de televigilancia ofrece nuevas oportunidades en el ajuste de la terapia, en conjunto con el personal de salud, para apoyar a los pacientes con DT2 ${ }^{20}$.

Por lo anterior se planteó como objetivo para realizar la revisión: identificar la evidencia de las intervenciones que evaluaron la efectividad de los mensajes de texto a través de teléfono móvil en la mejora de los niveles de $\mathrm{HbA1c}$.

\section{MÉTODOS}

La realización de la revisión se llevó a cabo en los meses de octubre y noviembre de 2012, estableciéndose inicialmente el plan de trabajo bajo el cual se desarrollaría metodológicamente el procedimiento. La revisión sistemática se realizó utilizando el modelo de siete pasos de Cooper $\left.^{13}: 1\right)$. Formulación del problema, para lo cual se estableció la pregunta de investigación: ¿Son útiles los mensajes de texto a través de telefonía inalámbrica en el control glucémico de los pacientes con DT2?; 2). Búsqueda de la literatura, que fue realizada de acuerdo a los criterios de selección definidos; 3 ). Recabar información de los estudios, para lo cual se seleccionaron aquellos considerados de interés para el propósito planteado 4). Evaluación de la calidad de los estudios, 5). Análisis e interpretación de los resultados de los estudios, 6). Interpretación de la evidencia, y 7). Presentación de resultados.

Los criterios de selección definidos para realizar la búsqueda establecieron como criterios de inclusión: artículos en idioma Inglés y Español publicados entre los años 2002 y 2012, que describieran la utilización de mensajes de texto mediante telefonía celular enviados a personas adultas con automanejo de DT2 para el control glucémico y reporte de HbA1c. Se excluyeron de la revisión los artículos de estudios que no fueran de intervención, que correspondían a otro tipo de diabetes, la utilización de otro tipo de tecnología de la información, como ordenadores, correo electrónico, o dispositivos electrónicos para el envío de mensajes de texto vía fax, y aquellas intervenciones diseñadas con finalidad diferente a la de monitorear los niveles de glicemia capilar o HbA1c.

Para la selección de información se realizó una búsqueda en Internet en las bases de datos Cochrane, CINAHL, Database of Abstracts of Reviews of Effectiveness (DARE), Academic Search Complete, MedicLatina, Open Access, MEDLINE, CENTRAL, ScienceDirect y Google Académico de los términos definitorios obtenidos en el Medical Subject Headings (MeSH). La estrategia de búsqueda se realizó a través del cruce de términos y palabras clave con el uso de operadores Booleanos AND, OR y NOT y los truncadores * $y$ " ". 
De las bases de datos consultadas se extrajeron los artículos que mencionaron en el título y el resumen los términos claves incluidos para la búsqueda. Para la selección de los artículos, las autoras efectuaron una lectura crítica de todos los resúmenes, se obtuvieron un total de 74 publicaciones. Después de revisar el título se excluyeron 19 artículos, y después de evaluar el resumen 35. De los 20 estudios resultantes se excluyeron 12 artículos, 5 por corresponder a otro tipo de tecnología, 2 por no corresponder el tipo de diseño, 2 que correspondieron a protocolos sin resultados, y 2 porque la muestra incluía a escolares con diabetes tipo 1 (DT1) y un estudio piloto que no incluyó medición de HbA1c.

Finalmente, para la revisión se contó con ocho estudios (Figura 1). Para asegurar la calidad de los estudios se utilizó la guía Critical Appraisal Skills Programe para ensayo clínico en Español (CASPe) ${ }^{21}$. Además para realizar el análisis de la información se contó con un formato elaborado en Microsoft Excel en el que se realizó el registro de los siguientes datos: Autor, revista, año de publicación, título del artículo, objetivo o propósito del estudio, lugar de realización, participantes, descripción de la intervención, duración de la intervención, hallazgos y resultados principales y conclusiones.

Figura 1. Diagrama de flujo del proceso de selección de los artículos para la revisión sistemática.

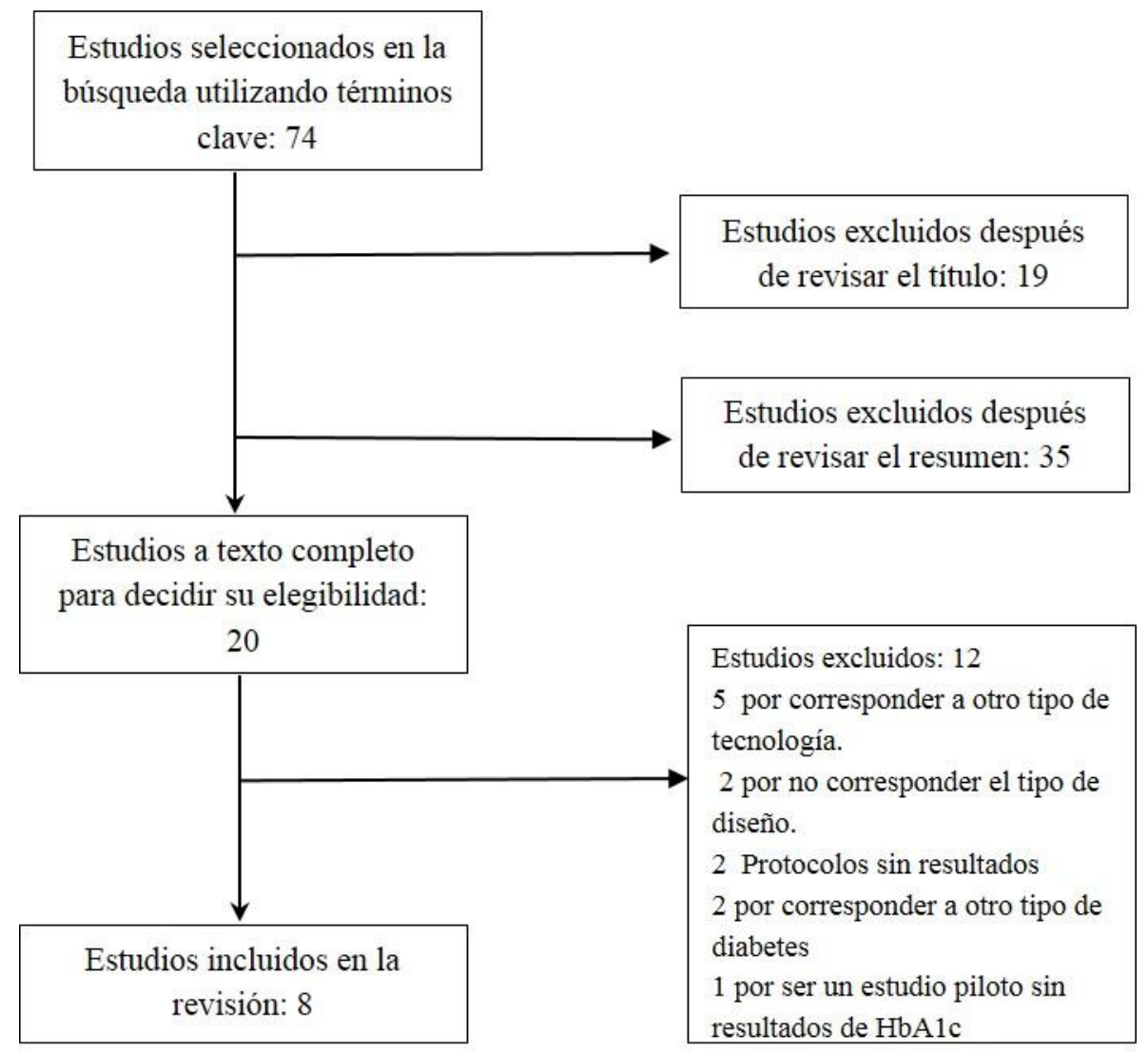

\section{RESULTADOS}

En la revisión proyectada se analizaron ocho estudios que cumplieron con los criterios de selección previamente establecidos. Los estudios incluidos fueron realizados 1 en Corea del Sur, 2 en Estados Unidos (USA), 1 en India, 2 en Irán y 2 en Reino Unido 
(UK). Cinco de ellos fueron ensayos clínicos aleatorizados, dos ensayos clínicos y dos estudios pre-test/post-test. La publicación de los mismos se realizó en diferentes revistas médicas especializadas en el cuidado de la diabetes principalmente y de telemedicina y telecuidados (Tabla 1).

Tabla 1. Artículos seleccionados para analizarse en la revisión

\begin{tabular}{|l|l|l|l|c|}
\hline \multicolumn{1}{|c|}{ Autor y año } & \multicolumn{1}{|c|}{ País } & \multicolumn{1}{c|}{ Diseño } & \multicolumn{1}{c|}{ Muestreo } & $\begin{array}{c}\text { Factor de impacto } \\
\text { de la revista }\end{array}$ \\
\hline Bell et al. 2012 & $\begin{array}{l}\text { Estados } \\
\text { Unidos }\end{array}$ & $\begin{array}{l}\text { Ensayo Clínico } \\
\text { Aleatorizado }\end{array}$ & $\begin{array}{l}\text { No } \\
\text { Probabilístico }\end{array}$ & 0.87 \\
\hline Goodarzi et al. 2012 & Iran & $\begin{array}{l}\text { Ensayo Clínico } \\
\text { Aleatorizado }\end{array}$ & Probabilístico & 4.39 \\
\hline Istepanian et al. 2009 & Reino Unido & $\begin{array}{l}\text { Ensayo Clínico } \\
\text { Aleatorizado }\end{array}$ & $\begin{array}{l}\text { No } \\
\text { Probabilístico }\end{array}$ & 1.467 \\
\hline Quinn et al. 2009 & $\begin{array}{l}\text { Estados } \\
\text { Unidos }\end{array}$ & $\begin{array}{l}\text { Ensayo Clínico } \\
\text { Aleatorizado }\end{array}$ & $\begin{array}{l}\text { No } \\
\text { Probabilístico }\end{array}$ & 1.93 \\
\hline Shetty et al. 2011 & India & Ensayo Clínico & $\begin{array}{l}\text { No } \\
\text { Probabilístico }\end{array}$ & 0.296 \\
\hline Turner et al. 2009 & Reino Unido & Ensayo Clínico & $\begin{array}{l}\text { No } \\
\text { Probabilístico }\end{array}$ & 0.503 \\
\hline Yoon et al. 2008 & $\begin{array}{l}\text { Corea del } \\
\text { Sur }\end{array}$ & $\begin{array}{l}\text { Estudio pre-test } \\
\text { /post-test }\end{array}$ & $\begin{array}{l}\text { No } \\
\text { Probabilístico }\end{array}$ & 2.61 \\
\hline Zolfaghari et al. 2012 & Iran & $\begin{array}{l}\text { Estudio pre-test } \\
\text { / post-test }\end{array}$ & $\begin{array}{l}\text { No } \\
\text { Probabilístico }\end{array}$ & 4.39 \\
\hline
\end{tabular}

La duración de las intervenciones osciló entre los tres y doce meses, el tamaño de muestra varió entre los 23 y los 215 participantes. Para la revisión se incluyeron los estudios de intervención que utilizaron el envío de mensajes de texto con información respecto al autocuidado, manejo de terapéutica prescrita, atención de dudas y vigilancia de niveles de glucosa sanguínea entre otros mensajes, a pacientes adultos con DT2. Los grupos controles recibieron atención de cuidado habitual proporcionada por el médico, enfermera especialista en diabetes o ambos de acuerdo al sistema médico del país en el consultorio, en el centro de atención de cuidado para la salud o en la clínica de diabetes.

Turner y colaboradores ${ }^{15}$ consideraron que el mayor logro en el automanejo fue que los pacientes recibieron mensajes educativos y de respuesta en tiempo real a los valores de glucosa en sangre reportados. Quinn y colaboradores ${ }^{17}$ destacaron por su parte que los pacientes se beneficiaron de la información sobre la diabetes, los medicamentos y los hábitos de vida comunicados a través de mensajes enviados a su teléfono móvil. En tanto que Yoon y $\mathrm{Kim}^{19}$ y Bell y colaboradores ${ }^{18}$ mencionaron que la intervención utilizando esta tecnología es simple de implementar y mantener considerándolo como un factor predictivo importante de éxito en el autocuidado para el control glucémico. Los hallazgos de Goodarzi y colaboradores ${ }^{22}$ demostraron la efectividad de la intervención utilizando SMS a través del teléfono móvil en el manejo de la DT2 destacando el uso de la telefonía celular para la educación a distancia de estos pacientes. Istepanian y colaboradores ${ }^{16}$ no encontraron diferencias en los niveles de $\mathrm{HbA1c}$ entre los grupos de intervención y control, describiendo que el envío de SMS no tiene una ventaja sobre el cuidado habitual, sin embargo la intervención tuvo una diferencia en la $\mathrm{HbA1c}$ a su favor de $0.64 \%(P=0.06)$. El estudio de Shetty y colaboradores $^{14}$ demostró que la comunicación a través de los SMS es bien aceptada por los pacientes con DT2 y contribuyo a mejorar los resultados de salud. Zolfaghari y 
colaboradores $^{23}$ destacaron que la utilización de SMS a través de teléfono celular mejoró los niveles de HbA1c y la adherencia al tratamiento prescrito en los pacientes con DT2 (Tabla 2).

Tabla 2. Resultados de la Intervención sobre los Niveles de HbA1c

\begin{tabular}{|c|c|c|c|}
\hline \multirow{2}{*}{ Autor y año } & \multicolumn{3}{|c|}{ Resultados de Niveles de HbA1c } \\
\hline & Antes & Después & \\
\hline Bell et al., (2012) & $\begin{array}{l}\text { Gl } 9.6-1.5 \\
D E^{-10} 9.0-0.09 D E\end{array}$ & $\begin{array}{l}-1.2 \\
-1.0\end{array}$ & $\begin{array}{l}\text { Discreto descenso del \% de } \\
\mathrm{HbA1c}\end{array}$ \\
\hline $\begin{array}{l}\text { Goodarzi et al. } \\
\text { (2012) }\end{array}$ & $\begin{array}{c}\text { GI } 7.91-1.24 D E \\
\text { GC } 7.83-1.12 \\
D E\end{array}$ & $\begin{array}{l}7.02-1.02 D E \\
7.8-1.26 D E\end{array}$ & $\begin{array}{l}\text { En el pre-test, no se } \\
\text { encontraron diferencias } \\
\text { significativas en la HbA1c entre } \\
\text { los grupos intervención y } \\
\text { control ( } p=0.227) \text {. }\end{array}$ \\
\hline $\begin{array}{l}\text { Istepanian et al., } \\
\text { (2009) }\end{array}$ & $\begin{array}{ll}\text { GI } 7.9-1.5 \\
\text { GC } 8.1-1.6\end{array}$ & $\begin{array}{l}7.76 \\
8.40\end{array}$ & $\begin{array}{l}\text { Al inicio no hubo diferencias en } \\
\text { la } \mathrm{HbA} 1 \mathrm{c} \text { entre los grupos de } \\
\text { control: } 7.9 \% \text { y } 8.2 \% \text {, } \\
\text { respectivamente }(P=0.17) \text {. De } \\
\text { los pacientes que completaron } \\
\text { el estudio, el grupo de } \\
\text { intervención tuvo una HbA1c } \\
0.64 \% \text { más baja que el grupo } \\
\text { control }(P=0.06) \text {. }\end{array}$ \\
\hline $\begin{array}{l}\text { Quinn et al., } \\
\text { (2009) }\end{array}$ & $\begin{array}{ll}\text { G1 } & 9.2 \pm 1.7 \\
\text { G2 } & 9.3 \pm 1.8 \\
\text { G3 } & 9.0 \pm 1.8 \\
\text { G4 } & 9.9 \pm 2.1 \\
\end{array}$ & $\begin{array}{l}8.5 \pm 1.8 \\
7.7 \pm 1.0 \\
7.9 \pm 1.4 \\
7.9 \pm 1.7\end{array}$ & $\begin{array}{l}\text { Mejora en el \% de Hb1Ac. } \\
\text { Decremento de un } 1.9 \% \text { (IC } \\
95 \% 1.5-2.3 \text { ) a los } 12 \text { meses }\end{array}$ \\
\hline $\begin{array}{l}\text { Shetty et al. } \\
\text { (2011) }\end{array}$ & $\begin{array}{l}\mathrm{Gl}->8 \mathrm{NE} \\
\mathrm{GC}->8 \mathrm{NE}\end{array}$ & $\begin{array}{l}8.9+1.4 \\
9.1+1.3\end{array}$ & $\begin{array}{l}\text { No hubo diferencias } \\
\text { significativas entre los grupos } \\
\text { intervención y control } \\
\text { (p<0.007). NE (No } \\
\text { Especificado) mencionado } \\
\text { únicamente como }>8 \%\end{array}$ \\
\hline $\begin{array}{l}\text { Turner et al., } \\
\text { (2009) }\end{array}$ & $9.5-2.2$ & 8.9 & $\begin{array}{l}\text { Mejora a los } 3 \text { meses de la } \\
\mathrm{Hb} 1 \mathrm{Ac} 0.52 \%(0.91)\end{array}$ \\
\hline Yoon, (2008) & $\begin{array}{ll}\text { GI } & 8.09 \pm 1.72 \\
\text { GC } & 7.59 \pm 1.09\end{array}$ & $\begin{array}{l}6.77 \pm 0.77 \\
8.40 \pm 1.04\end{array}$ & $\begin{array}{l}\text { En el pre-test, no se } \\
\text { encontraron diferencias } \\
\text { significativas en la HbA1c entre } \\
\text { los grupos. En el post-test los } \\
\text { niveles de HbA1c fueron } \\
\text { significativamente diferentes } \\
\text { entre los dos grupos ( } p= \\
0.001) \text {, y difirieron en el tiempo } \\
\text { (pre-test post-test frente) ( } p= \\
0.011 \text { ) }\end{array}$ \\
\hline
\end{tabular}

Seis de los ocho estudios incluidos mantuvieron contacto exclusivo a través de mensajes de texto, en uno se utilizó una llamada telefónica semanal ${ }^{17}$ del proveedor de cuidados al paciente si no tenía información de éste después de tres días de la fecha señalada para comunicar resultados, mientras que en otro estudio además se comparó el uso los SMS con las llamadas a teléfono ijo $^{23}$ (Tabla 3). 
Tabla 3. Características de las Intervenciones de los estudios seleccionados

\begin{tabular}{|c|c|c|c|c|c|c|c|}
\hline \multirow{3}{*}{ Autor $y$ atho } & \multirow{3}{*}{$\begin{array}{l}\text { Poblacion, } \\
\text { promedo de } \\
\text { edad, muestra }\end{array}$} & \multirow{3}{*}{$\begin{array}{l}\text { AAos con } \\
\text { diagnostloo de } \\
\text { DM }\end{array}$} & \multirow{3}{*}{$\begin{array}{l}\text { Duracion de } \\
\text { la Interven- } \\
\text { cion }\end{array}$} & \multicolumn{4}{|c|}{ Caracteristicas de il intervencion } \\
\hline & & & & \multicolumn{3}{|c|}{ Grupo Experimental } & \multirow{2}{*}{$\begin{array}{c}\text { Grupo } \\
\text { Control } \\
\text { Culdado habaual }\end{array}$} \\
\hline & & & & Envio SMS & Auto-manejo CG & Recomendaciones $\mathrm{CT}$ & \\
\hline Bell et al., (2012) & $\begin{array}{c}\text { Adultos } \\
58 \text { afीos } \\
54 \% \text { nombres } \\
n=65\end{array}$ & 13 & 12 meses & $\begin{array}{l}\text { SMS envio de videos } \\
\text { autoculdadoy } \\
\text { recordatorios }\end{array}$ & $\begin{array}{l}\text { Autoculoado } \\
\text { Monitoreo de glucosa }\end{array}$ & $\begin{array}{l}\text { Recomendaciones y } \\
\text { conbejos sobre el } \\
\text { culdado de la dlabetes, } \\
\text { apoyo para el estlo vida }\end{array}$ & $\begin{array}{l}\text { Tratamiento } \\
\text { eetablecido en } \\
\text { consulta medica }\end{array}$ \\
\hline $\begin{array}{l}\text { Goodarzl et al. } \\
\qquad(2012)\end{array}$ & $\begin{array}{c}\text { Aduitos } \\
53.8 \text { ahios } \\
77 \% \text { mujeres } \\
n=81\end{array}$ & Mas de un aho & 3 meses & $\begin{array}{l}4 \text { meneajes } \\
\text { educativos por } \\
\text { semana }\end{array}$ & $\begin{array}{l}\text { Dleta ejerciclo } \\
\text { medicamentos } \\
\text { importancla de auto } \\
\text { monitoreo de glucosa }\end{array}$ & $\begin{array}{l}\text { Sintomas de } \\
\text { hipoglicemia, mobvacion; } \\
\text { allmentacion } \\
\text { saludioles visita medica } \\
\text { C/2 meses }\end{array}$ & $\begin{array}{l}\text { Liamada telatonica } \\
\text { en la que no se } \\
\text { incluyo intormacion } \\
\text { educativa }\end{array}$ \\
\hline $\begin{array}{l}\text { Istepanlan et at. } \\
\text { (2009) }\end{array}$ & $\begin{array}{c}\text { Aduritos } \\
58.5 \text { a hos } \\
\pi=137\end{array}$ & 12.5 & 9 meses & $\begin{array}{l}\text { Envio de resultados } \\
\text { de glucosa capliar }\end{array}$ & $\begin{array}{l}\text { Automonitoreo de } \\
\text { glucosa en sangre } \\
\text { Envlo de resultados } \\
\text { desde el telafono movi }\end{array}$ & $\begin{array}{l}\text { Recibleron } \\
\text { recomendaciones sobre } \\
\text { al tratamiento y detalles } \\
\text { de } 106 \text { resultados }\end{array}$ & $\begin{array}{l}\text { Culdados por el } \\
\text { centro de dlabetes } \\
\text { o su medico local }\end{array}$ \\
\hline Quinn et al... (2009) & $\begin{array}{c}\text { Adultos } \\
53 \text { afios } \\
50 \% \text { mujeres } \\
n=163\end{array}$ & 7.9 & 12 meses & $\begin{array}{l}\text { MSM Motlvaclana| } \\
\text { En promedio una } \\
\text { Ilamada mensual }\end{array}$ & $\begin{array}{l}\text { Automonitoreo de } \\
\text { glucosa capllar }\end{array}$ & $\begin{array}{c}\text { Respuesta automatica } \\
\text { en tlempo real educativa, } \\
\text { conouctual y } \\
\text { motlvaclonal }\end{array}$ & $\begin{array}{l}\text { Acceso a pagina } \\
\text { web con } \\
\text { Informacion visual }\end{array}$ \\
\hline Snetty et al. 2011 & $\begin{array}{c}\text { Adultos } \\
50.5 \text { ah̆os } \\
n=215\end{array}$ & 5 & 12 meses & $\begin{array}{c}2 \text { mensajes } \\
\text { educativos por } \\
\text { semana sobre habios } \\
\text { saludables }\end{array}$ & $\begin{array}{l}\text { Registro diario de } \\
\text { alimentacion y actividad } \\
\text { flicica }\end{array}$ & 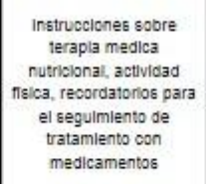 & $\begin{array}{l}\text { Consulta medica } \\
\text { Recilio programa } \\
\text { educativo sobre lo6 } \\
\text { medlcamentos y } \\
\text { camblo en el estlo } \\
\text { de vida }\end{array}$ \\
\hline Turner et al., (2009) & $\begin{array}{c}\text { Adultos } \\
57.6 \text { aौos } \\
78 \% \text { nombres } \\
n-23\end{array}$ & 6.4 & 12 meses & Alertas y recordatorlos & $\begin{array}{l}\text { Monitoreo de glucosa } \\
\text { a]uste de dosis de } \\
\text { Insulina }\end{array}$ & $\begin{array}{l}\text { Apoyo en la toma de } \\
\text { decisiones }\end{array}$ & $\begin{array}{l}\text { Consulta con } \\
\text { equipo de salud }\end{array}$ \\
\hline Yoon, (2008) & $\begin{array}{c}\text { Adurtos } \\
47 \text { aflos } \\
57 \% \text { mujeres } \\
n=51\end{array}$ & 6.6 & 12 meses & $\begin{array}{l}52 \text { sias en promedlo } \\
\text { para cada paclente } \\
\text { Mensajes de } \\
\text { Intormacion } \\
\text { motwacionales }\end{array}$ & Montsoreo de glucosa & $\begin{array}{c}\text { Recomendaciones } \\
\text { semanales sobre ia toma } \\
\text { de medicamentos orales } \\
\text { y aplicaclon de unidades } \\
\text { de insullina }\end{array}$ & $\begin{array}{c}\text { Consulta medlca } \\
\text { Se proporclonaron } \\
\text { recomendaciones } \\
\text { sobre los } \\
\text { medicamentos y } \\
\text { sus dosis y camblo } \\
\text { en el estlo de vida }\end{array}$ \\
\hline $\begin{array}{l}\text { Zolragharl, et al. } \\
\text { (2012) }\end{array}$ & $\begin{array}{c}\text { Adultos } \\
52.7 \text { ahios } \\
53 \% \text { mujeres } \\
n=77\end{array}$ & 7.1 & 3 meses & $\begin{array}{l}\text { A menos } 4 \text { SMS por } \\
\text { gemana educativos y } \\
\text { motivacionales }\end{array}$ & Montioreo de glucosa & $\begin{array}{c}\text { Consejeria sobre la } \\
\text { enfermedad, factores de } \\
\text { resgo, importancla de } \\
\text { mantener los niveles de } \\
\text { glucosa en rangos } \\
\text { normales }\end{array}$ & $\begin{array}{l}\text { Grupo de llamada } \\
\text { telefonlica } 2 \text { veces a } \\
\text { la semana el primer } \\
\text { mes posterlormente } \\
\text { Una vez al mes }\end{array}$ \\
\hline
\end{tabular}

\section{DISCUSIÓN}

La revisión realizada de los estudios de intervención basados en el uso de SMS a través de teléfono celular mostró una reducción significativa de los niveles de $\mathrm{HbA1c}$, sin embargo, no puede decirse que estos datos se deben a la utilización exclusiva de mensajes de texto para el automanejo. Se tiene que considerar que si bien es cierto se observó reducción significativa en seis de los ocho estudios de los niveles de $\mathrm{HbA} 1 \mathrm{c}$ la duración de las intervenciones no fue del mismo periodo de tiempo para todos los estudios. El control glicémico estuvo determinado por los niveles de HbA1c realizándose comparaciones al inicio y término de la intervención en ambos grupos experimental y control. Destacándose que los participantes del grupo de intervención de los ocho estudios obtuvieron cifras de niveles inferiores de $\mathrm{HbA} 1 \mathrm{c}$ al término de los mismos en comparación con el grupo control.

Istepanian, y colaboradores ${ }^{16}$ señalaron que no encontraron diferencias significativas en los niveles de HbA1c, entre el grupo experimental y el grupo control, datos que coinciden con resultados de estudios con otro tipo de intervención realizados en el Reino Unido como el de Farmer y colaboradores ${ }^{24}$ en el que señalaron que no se 
cuenta con evidencia convincente para incorporar los hallazgos en el autocuidado de pacientes con DT2 de que brindar apoyo intenso, mayor, o brindar solo cuidado habitual tenga efecto de mejora del control glucémico en personas con DT2 bien controladas. En dos de los estudios se utilizó apoyo mediante llamada telefónica ${ }^{17,23}$, uno utilizó video de cuidados $^{18}$ y los cinco restantes mencionaron el envío de mensajes de contenido educativo y motivacional; por lo anterior se infiere que no se contó con uniformidad entre las intervenciones que puedan demostrar que exclusivamente el envío de SMS es una estrategia efectiva para el control glucémico de los pacientes con DT2 al mantener o reducir las cifras de hemoglobina glucosilada iguales o menores a niveles de $7 \%$.

Cabe señalar que el personal de enfermería y los médicos que utilizaron por primera vez esta tecnología opinaron que tiene el potencial de mejorar la atención al paciente ${ }^{23}$. Contrario a lo descrito por Turner y colaboradores que destacaron la preocupación del personal médico que teme que las personas se vuelvan dependientes de la consulta y guía fuera de las instalaciones de cuidados de salud ${ }^{15}$.

Los rápidos avances en las tecnologías de la información y la comunicación abren nuevas posibilidades para el automanejo de la DT2. Los principales hallazgos demostraron que la intervención de consejería y/o asesoría basada en el uso de telefonía móvil utilizando SMS mejora los niveles de HbA1c. Cabe destacar que es necesario realizar más estudios sobre esta opción antes de que se generalice su utilización para la mayoría de las personas con DT2. Es innegable que los SMS utilizando el teléfono celular se asocian con la mejora del control glucémico esto es de particular interés debido a que actualmente las tasas de acceso a la telefonía celular son muy altas $^{12,17}$.

La información fiable y una comunicación eficaz son elementos cruciales en el cuidado de la salud. El uso de tecnologías apropiadas puede aumentar la calidad y el alcance tanto de la información como de la comunicación. Por un lado, la información es la base del conocimiento que permite a las personas mantener su propia salud, y por el otro, las organizaciones sociales ayudan a las personas a alcanzar la salud a través de los sistemas de atención para la salud. ${ }^{25}$

\section{CONCLUSIÓN}

Se concluye que los SMS comprenden una nueva tecnología de comunicación que permite a una persona con un teléfono móvil enviar un mensaje de texto a otro teléfono móvil. Los SMS se envían casi instantáneamente al teléfono móvil de destino y pueden leerse casi en cualquier lugar lo que es muy conveniente para el receptor.

Es además relativamente barato, eficiente, cómodo y menos intrusivo en comparación con una llamada de teléfono. En la actualidad su disponibilidad es muy amplia y cada vez es más utilizado alrededor del mundo. La relativa facilidad con la que puede ser enviado un SMS favorece el intercambio de información entre el paciente con DT2 y el personal de salud además de mantener comunicación continua.

En los últimos años varias aplicaciones de la tecnología de la información basadas principalmente en la telefonía móvil se han desarrollado con el fin de ayudar a los pacientes a manejar fuera de la clínica condiciones crónicas como la DT2. Sin embargo el escaso número de artículos incluidos se considera una limitante de la presente revisión al no encontrarse al momento de su realización de una mayor 
aportación de evidencia para señalar resultados concluyentes sobre la mejora del control glucémico a través de esta tecnología de la información.

\section{REFERENCIAS}

1. International Diabetes Federation. (2011). IDF Diabetes Atlas, 5th edn. Brussels, Belgium: International Diabetes Federation. Disponible en: http://www.idf.org/diabetesatlas. Consultado Febrero, 2013.

2. World Health Organization. The diabetes program 2012. Disponible en: http//www.who.int/diabetes/en/. Consultado Abril, 2013.

3. Secretaria de Salud. Encuesta nacional de salud y nutrición 2012. Instituto Nacional de Salud Pública. Subsecretaria de Prevención y Promoción de la Salud. 2012. recuperado de: http://ensanut.insp.mx/

4. Aguilar C, Gómez R, Gómez FJ. La diabetes tipo 2 en México: principales retos y posibles soluciones. Revista de la ALAD. 2011; 19(4):148-161.

5. Villalpando S, Rojas R, Shamah-Levy T, Ávila M, Gaona B, De la Cruz V, Rebollar R, Hernández L. Prevalence and distribution of type 2 diabetes mellitus in Mexican adult population: a probabilistic survey. Revista de Salud Pública de México. 2010; 52(IS): 19-26.

6. Hoyos T, Arteaga M, \& Muñoz M. (2011). Factores de no adherencia al tratamiento en personas con Diabetes Mellitus tipo 2 en el domicilio. La visión del cuidador familiar. Revista de Investigación y Educación en Enfermería; 29(2):194-203.

7. American Diabetes Association. Standards of medical care in diabetes-2013. Diabetes Care. 2013; 36(1S): 11-66.

8. Chen L, Magliano D, Zimmet P. The worldwide epidemiology of type 2 diabetes mellitus: present and future perspectives. Nature Reviews, Endocrinology. 2012; 8: 228-236.

9. Lawrenson R, Joshy G, Eerens Y, Johnstone W. How do newly diagnosed patients with type 2 diabetes in the Waikato get their diabetes education? Journal of Primary Health Care. 2010; 2(4):303-310.

10. Bravo-Toledo R. Nuevas tecnologías. Actualización en Medicina Familiar. 2011; 7(6):356-357.

11. Alfaro M, Bonisb J, Bravo R, Fluitersd E, Minuée S. Nuevas tecnologías en atención primaria: personas, máquinas, historias y redes. Gaceta Sanitaria. 2012; 26(S):107-112.

12. Fischer $H$, Moore S, Ginosar D, Davidson A, Rice-Peterson C, Durfee $M$, Mackenzie T, Estacio R, Steele A. Care by cell phone: text messaging for chronic disease management. American Journal of Managerial Care. 2012; 18(2): $42-47$.

13. Cooper H. (2010). Research synthesis and meta-analysis: a step by step approach (4th ed). SAGE Publications, Inc. thousand Oaks, California.

14. Shetty AS., Chamukuttan S., Nanditha A., Champat RK., Ramachandran A. Reinforcement of adherence to prescription recommendations in Asian Indian diabetes patients using short message service (sms)-a pilot study. Journal of the Association of Physicians of India. 2011; 59.

15.Turner J., Larsen M., Tarassenko L., Neil A., Farmer A. Implementation of telehealth support for patients with type 2 diabetes using insulin treatment: an exploratory study. Informatics in Primary Care. 2009;17:47-53. 
16. Istepanian K, Harry D, Moutosammy N, Sungoor A, Tang B, Earle KA. Evaluation of a mobile phone telemonitoring system for glycemic control in patients with diabetes Journal of Telemedecine and Telecare. 2009; 15:125128.

17.Quinn CC., Gruber-Baldini AL., Shardell M., Weed K., Clough SS., Peeples M., Terrin M., Bronich-HallL., Barr E., Lender D. Mobile diabetes intervention study: testing a personalized treatment/behavioral communication intervention for blood glucose control. Contemporary Clinical Trials. 2009; 30(4): 334-46.

18. Yoon KH, Kim HS. A short message service by cellular phone in type 2 diabetic patients for 12 months. Diabetes Research and Clinical Practice. 2008; 79:256261.

19.Bell AM., Fonda SJ., Walker MS., Schmidt V., Vigersky RA. Mobile phonebased video messages for diabetes self-care support. Journal of Diabetes Science and Technology. 2012; 6(2):310-319.

20.Chang, K., Davis, R., Birt, J., Castelluccio, P., Woodbridge, P., Marrero, D. Nurse practitioner-based diabetes care management impact of telehealth or telephone intervention on glycemic control. Manage Health Outcomes. 2007; 15(6): 377-385.

21. Institute of Health Sciences of Oxford University (2008). Critical Appraisal Skills Programme Español-CASPe. Boston, MA: Oxford University. Disponible en: http://www.redcaspe.org/herramientas/index.htm

22. Goodarzi M., Ebrahimzadeh I, Rabi A, Saedipoor B, Jafarabadi MA. Impact of distance education via mobile phone text messaging on knowledge, attitude, practice and self-efficacy of patients with type 2 diabetes mellitus in Iran. Journal of Diabetes \& Metabolic Disorders. 2012; 11(10).

23.Zolfaghari M., Mousavifar SA., Haghani $\mathrm{H}$. Mobile phone text messaging and telephone follow-up in type 2 diabetic patients for 3 months: a comparative study. Journal of Diabetes \& Metabolic Disorders. 2012; 11(7).

24.Farmer A., Wade A., Goyder E., Yudkin P., French D., Craven A., Holman R., Kinmonth A-L., Neil A. Impact of self-monitoring of blood glucose in the management of patients with non-insulin treated diabetes: open parallel group randomized trial. British Medical Journal. 2007; July 21;335:132

25. Chetley, A., Davies, J., Trude, B., McConnell, H., Ramirez, R. Improving health, connecting people: the role of ICTs in the health sector of developing countries. Washington, D.C., World Bank, Information for Development Program. 2006 May 31. (25).

Recibido: 12 diciembre 2013; Aceptado: 26 enero 2014

ISSN 1695-6141

๑ COPYRIGHT Servicio de Publicaciones - Universidad de Murcia 